\title{
BMJ open Children, smoking households and exposure to second-hand smoke in the home in rural Australia: analysis of a national cross-sectional survey
}

\author{
Jo M Longman, Megan E Passey
}

To cite: Longman JM, Passey ME. Children, smoking households and exposure to second-hand smoke in the home in rural Australia: analysis of a national cross-sectional survey. BMJ Open 2013;3: e003128. doi:10.1136/ bmjopen-2013-003128

- Prepublication history for this paper is available online. To view these files please visit the journal online (http://dx.doi.org/10.1136/ bmjopen-2013-003128).

Received 26 April 2013 Revised 4 June 2013 Accepted 12 June 2013

This final article is available for use under the terms of the Creative Commons Attribution Non-Commercial 3.0 Licence; see http://bmjopen.bmj.com

University Centre for Rural Health, University of Sydney School of Public Health, University of Sydney, Lismore, New South Wales, Australia

\section{Correspondence to} Dr Jo M Longman; jo.longman@ucrh.edu.au

\begin{abstract}
Objectives: This paper aimed to explore the association between rurality and (1) household smoking status and (2) home second-hand smoke exposure, in households with children aged 0-14 years.

Design: Cross-sectional study.

Setting: Households across Australia.

Participants: Households across the country were randomly selected to provide a nationally representative sample. Respondents were persons aged 12 years or older in each household who were next going to celebrate their birthday.
\end{abstract}

Primary outcome measures: Household smoking status and smoking inside the home.

Methodology: The 2010 Australian National Drug Strategy Household survey data were analysed to explore the prevalence of household smoking and home secondhand smoke exposure in rural and urban households with children. Multivariable logistic regression was used to explore the association of rurality with household smoking and with home second-hand smoke exposure, controlling for potential confounders.

Results: Households with children were more likely to be smoking households $(35.4 \%, 95 \% \mathrm{Cl} 34.2 \%$ to $36.5 \%)$ than households without children $(32.1 \%, 95 \%$ $\mathrm{Cl} 31.3 \%$ to $32.8 \%$ ). Both household smoking (43.6\% (95\% Cl $41.5 \%$ to $45.7 \%$ ) vs $31.4 \%(95 \% \mathrm{Cl} 30.0 \%$ to $32.8 \%)$ ) and home second-hand smoke exposure (8.0\% $(95 \% \mathrm{Cl} 6.8 \%$ to $9.1 \%$ ) vs $5.2 \%$ ( $95 \% \mathrm{Cl} 4.5 \%$ to $5.8 \%)$ ) were significantly more common for rural children. In multivariate analyses controlling for confounding factors, rurality remained associated with smoking households (OR 1.21, 95\% $\mathrm{Cl} 1.07$ to 1.37 ), whereas it did not remain associated with children's home second-hand smoke exposure (OR 1.07, $95 \% \mathrm{Cl}$ 0.85 to 1.35). Larger household size, low socioeconomic status and being a single-parent household were the main drivers of home second-hand smoke exposure.

Conclusions: The proportion of smoking households with children, and the number of children regularly exposed to second-hand smoke in their homes remain important public health concerns. Smoking cessation support and tobacco control policies might benefit from targeting larger and/or socioeconomically disadvantaged households including single-parent households.

\section{ARTICLE SUMMARY}

Article focus

- Given that children's exposure to second-hand smoke is such an important public health concern, as well as the higher prevalence of smoking in rural areas in Australia, this paper addresses two questions: (1) Are there rural/ urban differences in (i) smoking within households with children and (ii) home second-hand smoke exposure in children? (2) If so, are these differences due to socioeconomic disadvantages or other factors unique to 'rurality'?

\section{Key messages}

- $43.6 \%$ of rural households with children contained a smoker. Both household smoking and home second-hand smoke exposure were significantly more common for rural children than for urban dwelling children. Rurality remained associated with smoking households but did not remain associated with home second-hand smoke exposure when confounding factors were controlled for. Children's home second-hand smoke exposure was driven by larger household size, low socioeconomic status and being a single-parent household.

Strengths and limitations of this study

- No previous studies have explored rural/urban differences in smoking in households with children in Australia. This study used data from a representative national household survey to explore the association of rurality with household smoking and with home second-hand smoke exposure. The study had limited potential household-level confounding variables available for inclusion in the analysis, and was limited by potential response bias leading to a possible under-reporting of household smoking status.

\section{BACKGROUND}

The negative impact of children's exposure to smoking behaviour and to second-hand smoke in their homes is extensive. Owing to 
the higher breathing rates per body weight, and the size and immaturity of their organs, children are particularly vulnerable to the effects of second-hand smoke exposure on their physical health. ${ }^{1-3}$ They are also particularly vulnerable to the corollaries of those effects, such as missing more time at school, and compromised academic performance. ${ }^{3}$ In addition, children whose parents smoke are more likely to become smokers themselves, growing up with smoking as a normalised behaviour. ${ }^{45}$

The site where most second-hand smoke exposure in children happens is their home. ${ }^{2}{ }^{6}$ Children are not able to control their environment to minimise their exposure and are effectively 'trapped' in smoking environments. Children who live in homes where there is no indoor smoking are afforded some protection from second-hand smoke exposure even when a parent smokes. ${ }^{6}{ }^{7}$ However, for adults, negotiating smoke-free home environments can be challenging due to the social and familial relationship aspects of smoking behaviour in the home. ${ }^{28}$

While it may be considered an infringement of individual civil liberties to legislate against second-hand smoke exposure in the home, the notion of a smoke-free home is an extension of the protection offered from comprehensive smoke-free legislation. In Australia, this began in the mid-1980 s, and has covered public transport, the workplace, public spaces, smoking in cars containing children, and, most recently, proposed legislation on multiunit dwellings, supported by an active campaign from Action on Smoking and Health Australia. ${ }^{3}$

This legislative environment reflects the importance of protecting the public from second-hand smoke exposure. Efforts to protect children from the effects of second-hand smoke exposure respond directly to article 8 of the WHO Framework Convention on Tobacco Control requiring smoke-free measures by those countries ratifying the treaty, including Australia. ${ }^{9}$

Educational campaigns focusing on smoke-free homes have been in place since the early 2000s and continue to form a regular part of tobacco campaigning in Australia. These campaigns have promoted quitting or smoking outside to protect children from the harms of home second-hand smoke exposure ${ }^{10}$ and appear to have been 'moderately helpful' in increasing the number of smoke-free homes. ${ }^{3}$ In 2006, 43\% of Australian households with smokers reported totally banning smoking indoors, ${ }^{11}$ and data from both New South Wales and Victoria show declines in household indoor smoking over recent years. ${ }^{3}$ One of the main outcome indicators in Australia's draft National Tobacco Strategy 2004-2009 is that fewer people will be exposed to second-hand smoke in their homes. ${ }^{12}$

\section{Is there an urban/rural divide in children's exposure to home second-hand smoke?}

In Australia, around one-third of the population lives outside major cities, with rural areas being more socioeconomically disadvantaged than urban areas. ${ }^{13}$ The prevalence of tobacco smoking in this rural population is significantly higher than in urban areas. ${ }^{14}$ Smoking continues to be disproportionately represented among socioeconomically disadvantaged populations, and these disparities are increasing over time. ${ }^{15}$

We identified only three studies exploring the difference between home second-hand smoke exposure in children in rural compared with urban areas, ${ }^{16-18}$ with none from Australia. Two are North American (one showing higher home second-hand smoke exposure in rural areas ${ }^{18}$ and one from Alaska showing lower home second-hand smoke exposure in very remote areas ${ }^{17}$ ), and one is from Germany reporting lower home secondhand smoke exposure in children in rural areas. ${ }^{16}$ One study from China describes second-hand smoke exposure in respondents' homes (all homes, not just homes with children) that is higher in rural areas $(73 \%)$ compared with urban areas $(60 \%) .{ }^{19}$ One further North American study focuses on home smoking bans, and reports fewer home smoking bans in rural households compared with urban households. ${ }^{20}$

Given that second-hand smoke exposure is such an important public health concern, particularly in children, as well as the higher prevalence of smoking in rural areas in Australia, this paper addresses two questions: (1) Are there rural/urban differences in (i) smoking within households with children and (ii) home second-hand smoke exposure in children? (2) If so, are these differences due to socioeconomic disadvantage or other factors unique to 'rurality'?

\section{METHODS}

\section{The 2010 National Drug Strategy Household Survey}

The Australian Institute of Health and Welfare (AIHW) conducts the National Drug Strategy Household Survey (NDSHS) every 3 years to measure behaviour, knowledge and attitudes towards drugs in Australia. The 2010 sample was a multistage random sample of households stratified by region with some oversampling in certain states and territories, representative of the national population. Respondents were persons aged 12 years or older in each household who were next going to celebrate their birthday. Questions were asked via drop and collect self-completion questionnaires. Questions were asked of the respondent about themselves, for example, their own smoking status, as well as questions about the household. Data were collected between April and September 2010. The survey has been conducted on nine previous occasions. ${ }^{21}$

NDSHS was chosen because it provided data on household behaviour, rather than just individual behaviour, as well as the number and age of children within a household. Non-identifiable data were sourced from AIHW with a signed agreement to protect the confidentiality and management of the data. 


\section{Weighting}

Weights were applied to the data to adjust for the complex sampling design while not inflating the overall sample size. Weights for each household record were provided by AIHW to adjust the number of households in the dataset to the number of households in the Australian population. Applying these rates would have overinflated the sample size for statistical analyses. Following Gartner and $\mathrm{Hall}^{15}{ }^{15}$ we proportionally adjusted the absolute weight for households provided by AIHW in order to bring the overall number of households down to the effective sample size. Each weight provided by AIHW was multiplied by an adjustment factor and then applied to the data to bring the total number of households down to the effective sample size provided by AIHW.

\section{Measures}

Rurality

The rurality variable was based on the Australian Standard Geographical Classification remoteness areas. $^{22}$ This classification was designed to facilitate quantitative comparison between 'city' and 'country' Australia, and is based on distance to services and goods. The variable was coded: major cities, inner regional, outer regional and remote/very remote. The inner regional code includes locations close to major cities, for example, Port Stephens, an hour's drive away from Newcastle (a major city), and outer regional includes locations such as Biloela in Queensland, $550 \mathrm{~km}$ away from the nearest major city (Brisbane). For more information, please see the map of Australia at http://www.abs.gov.au/websitedbs/D3310114.nsf/

home/remoteness+structure\#Anchor2e illustrating the remoteness structure. This classification system is recommended by the Australian Government's Institute of Health and Welfare (a statutory body established to report to the nation on health), particularly for use at the aggregated national level, ${ }^{23}$ and is widely used in the academic literature, for example, Kelly et $a l^{24} \mathrm{We}$ recoded the variable into urban/rural where urban was 'major cities' and rural was a combination of the remaining categories, as some cells contained small numbers with the initial categorisation.

\section{Households with children}

We defined households with children as those with children $0-14$ years old. The questionnaire asked "Of all the dependent children, how many are in each of these age categories?" ${ }^{25}$ and offered 2-year blocks up to 1214 years with the last category being $15+$. As "dependent children" was defined to include children older than 14 "who are still financially dependent, such as full-time students," ${ }^{25}$ we did not include the final category which may have contained substantial numbers of dependent children over 18 years.
Outcome variables

\section{Smoking households}

We defined smoking households as any household containing a smoker regardless of where that person smoked, that is, answering "Yes, inside the home" or "No, only smoke outside the home" to the question "In the last 12 months, have you or any other member of your household smoked at least one cigarette, cigar or pipe of tobacco per day in the home?" The other response option was "No-one at home regularly smokes." 25

\section{Home second-hand smoke exposure}

Home second-hand smoke exposure is different from smoking households as it is a measure of whether a household contains a smoker who smokes inside the home. This was determined as those households where the answer "Yes, inside the home" was given to the question "In the last 12 months, have you or any other member of your household smoked at least one cigarette, cigar or pipe of tobacco per day in the home?"25 We recoded this into a binary variable: "yes smokes inside the home" or "no does not smoke inside the home." The "no does not smoke inside the home" category included households answering either "No, only smokes outside the home" or "no-one at home regularly smokes."

\section{Potential confounders}

The data contained limited household-level variables. Within these confines, the following variables were considered as potential confounders in the association between home second-hand smoke exposure and rurality: socioeconomic status (SES), ${ }^{2}{ }^{15}$ number of people in the household, ${ }^{15}$ household structure ${ }^{316}$ and main language used in the household. ${ }^{3}$ These are defined below.

\section{Socioeconomic status}

We defined SES using the area-level socioeconomic index for areas (SEIFA) provided in the data (the index of relative socioeconomic advantage and disadvantage, measured at Census Collection District areas). ${ }^{26}$ This index includes variables on: income, education, employment, occupation, housing and other variables such as disability, car ownership, internet access and single parents.

\section{Household size}

The number of people in the household was recoded from $1,2,3,4,5$ or more to three categories: $1-2,3-4$ and 5 or more, as some cells contained small numbers with the initial categorisation.

\section{Household structure}

Household structure was coded as single with dependants, couple with dependants, parents with nondependent children, singles without children, couple without children and other. We recoded this to single 
with dependants, couple with dependants and other (included all other categories).

\section{Main language used in the household}

We maintained the original binary coding for this variable of English and for a language other than English.

\section{Analysis}

Analyses were performed using Stata V.9.2. Records with missing data on household smoking status were excluded from the analysis (31 households with children aged $0-14$ years). Summary statistics of household characteristics were obtained and univariate associations with rurality were examined using Pearson's $\chi^{2}$ test. Initial analyses included all households with data on smoking status. Subsequent analyses were restricted to households with children aged 0-14 years. Multivariable logistic regression was used to explore the association of rurality with household smoking and with home second-hand smoke exposure, controlling for potential confounders. Initially, all variables with $\mathrm{p}<0.25$ in the univariate analyses were included in the models, with stepwise removal of variables based on the $\mathrm{p}$ value from the likelihood ratio test, with variables with $\mathrm{p}<0.1$ retained in the model. Rurality was retained in both multivariable models regardless of statistical significance.

\section{RESULTS}

\section{Sample}

Weighting the sample to take account of the complex sampling $^{15}$ gave a total sample size of 15978 households (the effective sample size). Household smoking status was recorded for 15745 of these households, which were included in the analysis.

In all households, that is, those with children and those without children (33\%, 95\% CI $32.4 \%$ to $33.7 \%$ ) contained a smoker. Rural households were significantly more likely to contain a smoker than urban households (36.5\% (95\% CI $35.4 \%$ to $37.5 \%)$ vs $31.4 \%$ (95\% CI $30.6 \%$ to $32.1 \%)$ ), and were significantly more likely to contain a smoker who smoked indoors (home secondhand smoke exposure; $11.7 \%$ (95\% CI $11.0 \%$ to $12.5 \%$ ) vs $9.2 \%$ (95\% CI $8.7 \%$ to $9.6 \%)$ ). Households with children aged 0-14 years were more likely to be smoking households (35.4\%, 95\% CI $34.2 \%$ to $36.5 \%$ ) than households without children $(32.1 \%$, $95 \%$ CI $31.3 \%$ to $32.8 \%$ ).

For all subsequent reported analyses, we selected only those households with children aged $0-14$ years. This provided a sample of 4669 households.

\section{Smoking households with children}

Table 1 presents the demographic and smoking behaviour characteristics of households with children aged $0-14$ years.

Overall, $35.4 \%$ (95\% CI $34.2 \%$ to $36.5 \%$ ) of households with children were smoking households, and $6.1 \%$

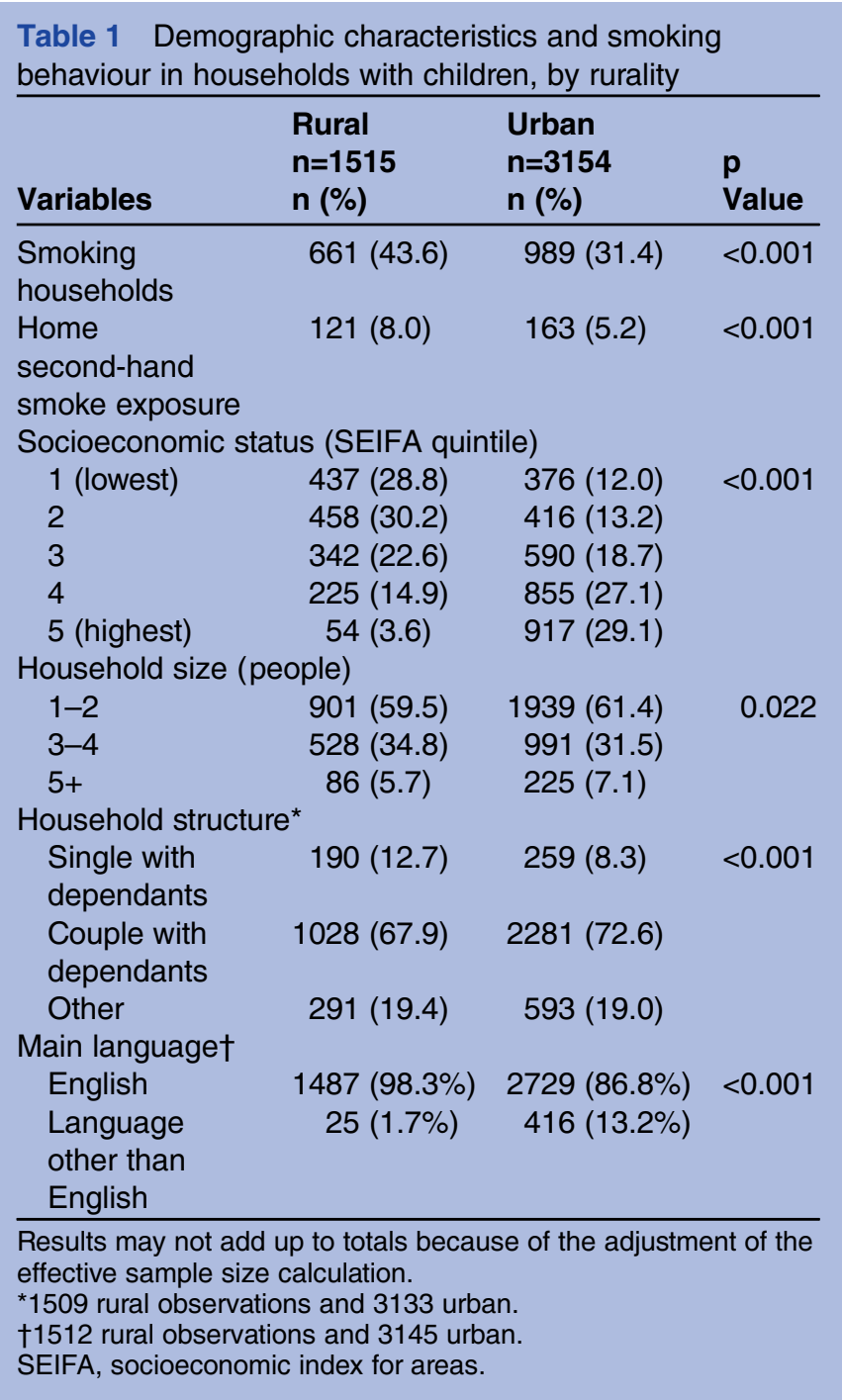

(95\% CI $5.5 \%$ to $6.7 \%)$ of households with children contained a smoker who smoked inside the home (home second-hand smoke exposure). The proportion of households which were smoking households was greater in rural $(43.6 \%, 95 \%$ CI $41.5 \%$ to $45.7 \%)$ than urban $(31.4 \%, 95 \%$ CI $30.0 \%$ to $32.8 \%)$ areas. The proportion of households with home second-hand smoke exposure was also significantly greater in rural $(8.0 \%$, $95 \%$ CI $6.8 \%$ to $9.1 \%)$ than urban $(5.2 \%, 95 \%$ CI $4.5 \%$ to $5.8 \%$ ) households with children.

Rural and urban households were also different on all potential confounders: the proportion of households in each SEIFA quintile $(p \leq 0.001)$; household size, with rural households having a smaller proportion of larger households $(p=0.022)$; household structure, with rural households having a larger proportion of households with a single adult with dependent children $(p \leq 0.001)$; and main language with a much lower proportion of rural households speaking a language other than English $(p \leq 0.001)$ than urban households. 
Associations of smoking behaviour with rurality Household smoking

Table 2 reports the results of the logistic regression model exploring the association of rurality with smoking households among households with children aged 014 years, controlling for potential confounders.

Among households with children, rurality continued to be associated with being a smoking household when SES, household size and household structure were controlled for. The main language used in the household was not retained in the model.

\section{Home second-hand smoke exposure}

Results of the logistic regression model to explore the association between rurality and home second-hand smoke exposure in households with children, controlling for potential confounders, are shown in table 3.

In households with children, home second-hand smoke exposure was not significantly associated with rurality once other factors were controlled for. SES, household size and household structure remained strongly associated with home second-hand smoke exposure. The main language used in the household was not retained in the model.

\section{DISCUSSION}

One-third of all Australian households were smoking households. In univariate analysis, both smoking households and home second-hand smoke exposure were significantly more common for rural households with children than for urban households with children. In a multivariate analysis among households with children, rurality remained associated with smoking households when

Table 2 Logistic regression model of factors significantly associated with smoking households among households with children

\begin{tabular}{ll}
\hline Variables & OR $(95 \%$ Cl) \\
\hline Rurality & \\
$\quad$ Urban & 1.00 \\
$\quad$ Rural & $1.21(1.07$ to 1.37$)$ \\
Socioeconomic status (SEIFA quintile) & \\
1 (lowest) & 1.00 \\
2 & $0.70(0.59$ to 0.84$)$ \\
3 & $0.56(0.47$ to 0.67$)$ \\
4 & $0.47(0.40$ to 0.57$)$ \\
5 (highest) & $0.28(0.23$ to 0.34$)$ \\
Household size (people) & \\
$1-2$ & 1.00 \\
$3-4$ & $1.20(1.06$ to 1.36$)$ \\
$5+$ & $1.36(1.08$ to 1.72$)$ \\
Household structure & \\
Single with dependants & 1.00 \\
Couple with dependants & $0.47(0.40$ to 0.56$)$ \\
$\quad$ Other & $0.70(0.56$ to 0.87$)$ \\
\hline SEIFA, socioeconomic index for areas. &
\end{tabular}

Table 3 Logistic regression model of factors associated with home second-hand smoke exposure among households with children

\begin{tabular}{ll}
\hline Variables & OR $(95 \%$ Cl) \\
\hline $\begin{array}{l}\text { Rurality } \\
\text { Urban }\end{array}$ & 1.00 \\
$\quad$ Rural & $1.07(0.85$ to 1.35$)$ \\
Socioeconomic status (SEIFA quintile) & \\
1 (lowest) & 1.00 \\
2 & $0.55(0.41$ to 0.74$)$ \\
3 & $0.52(0.38$ to 0.70$)$ \\
4 & $0.27(0.19$ to 0.38$)$ \\
5 (highest) & $0.25(0.17$ to 0.37$)$ \\
Household size (people) & 1.00 \\
$1-2$ & $1.83(1.42$ to 2.35$)$ \\
$3-4$ & $2.71(1.84$ to 4.00$)$ \\
$5+$ & 1.00 \\
Household structure & $0.25(0.19$ to 0.33$)$ \\
$\quad$ Single with dependants & $0.52(0.38$ to 0.73$)$ \\
Couple with dependants & \\
Other & \\
\hline SEIFA, socioeconomic index for areas. &
\end{tabular}

confounding factors were controlled for, whereas rurality did not remain associated with home second-hand smoke exposure after controlling for potential confounders.

\section{Smoking households}

Overall, smoking prevalence is in decline in Australia, estimated at $15.9 \%$ of adults smoking daily in $2010 .{ }^{3}$ Given this, it is surprising that $33 \%$ of all Australian households contain a daily smoker. Smoking is even more common in households containing children, with $35.4 \%$ of households with children aged $0-14$ years containing a smoker, rising to $43.6 \%$ of rural households with children $(\mathrm{p} \leq 0.001)$.

It is possible that a focus on individual smoking behaviour and not on household behaviours, children and/or rurality has masked recognition of this high proportion of rural households with children containing a smoker. The exploration of smoking at the household-level is supported by the importance of the social and relational environment for smoking, and smoking cessation. ${ }^{27}$

In households with children, rurality continued to be associated with smoking households when other factors were controlled for. This suggests that there might be something particular about rural smoking households that warrants further investigation, and that support for smoking cessation might be rurally targeted.

\section{Home second-hand smoke exposure}

Home second-hand smoke exposure was significantly more common for rural $(8 \%)$ than urban $(5.2 \% \mathrm{p} \leq$ 0.001 ) households with children. Using the NDSHS data, this equates to 54123 rural households with at least one child containing a daily smoker who smokes inside the home. The few studies of children's home second-hand 
smoke exposure in rural compared with urban areas provide a mixed picture, and comparison is compromised by differences in defining home second-hand smoke exposure. A German study ${ }^{16}$ exploring ruralurban comparisons of home second-hand smoke in households with children reported higher levels of home second-hand smoke in urban areas. This probably reflects the higher prevalence of smoking in urban than rural areas in European countries, ${ }^{28}$ the reverse of Australia, Canada and China. One North American study of states containing the Appalachian counties used a definition of home second-hand smoke exposure similar to our own, and reports rural home second-hand smoke exposure of children (under 18) at $12.5 \%$ compared to urban exposure at $6.5 \% .^{18}$ In contrast, urban Alaskan households have higher levels of home second-hand smoke exposure than the most extremely rural regions. ${ }^{17}$

The main drivers of home second-hand smoke exposure for children in rural households were the same as for urban, that is, low SES, larger household size and being a single parent household. Home second-hand smoke exposure is highest for children from disadvantaged families as their parents are more likely to smoke and smoke heavily, ${ }^{2}$ and to smoke in the home. ${ }^{16}$ The NDSHS data highlight the stark contrast between rural and urban households in terms of their SES, with only $3.6 \%$ of rural households in the least disadvantaged SEIFA category compared to $29.1 \%$ of urban households.

Another factor driving the higher prevalence of home second-hand smoke exposure in rural households compared with urban households is the significantly greater proportion of single-parent families in rural areas. The finding of higher home second-hand smoke exposure among single-parent families has been reported in Australia previously. ${ }^{29}$ Given that single parenthood is closely intertwined with disadvantage, ${ }^{3}$ we must continue with efforts to address smoking in lower SES populations groups.

Our finding that home second-hand smoke does not remain associated with rurality once confounders are adjusted for matches the Alaskan study described earlier. ${ }^{17}$ While rurality does not remain associated with home second-hand smoke exposure in households with children once other factors are controlled for, rural children experience more home second-hand smoke exposure because of these factors which place them at additional disadvantage (low SES and single-parent households). SES and household structure were the strongest drivers of both household smoking and home second-hand smoke.

Given the progressive adoption of smoke-free public places legislation in Australia and other high income countries, the relative importance of home second-hand smoke exposure is increasing. As living in a smoke-free home offers children some protection from home second-hand smoke exposure, continuing the decline in homes with smokers who smoke inside the house is key. However, one Swedish study of young children demonstrated that 'smoking outdoors' even with the door shut resulted in higher urinary cotinine levels when compared with an age-matched non-smoking household control group. ${ }^{7}$ These findings are supported by a UK study which reported that children from smoking parents in households which were smoke free had less salivary cotinine than children with smoking parents in households which were not smoke free, but more than children with non-smoking parents in smoke-free households. ${ }^{6}$ Therefore, while living in a household with no indoor smoking offers children some protection, children from households without smokers are the most protected.

The data explored in this study did not include children's second-hand smoke exposure outside their own home, for example, in cars, visiting other families, social outings, etc but as rural children are more likely to live in a smoking household (even if the smoker/s smoke outside the home), we would anticipate the likelihood that they are also more frequently exposed to secondhand smoke in other non-home settings. In a study of infants in urban New South Wales, Australia, for example, Daly et $a l^{29}$ report that $10 \%$ were exposed in the home, and $22 \%$ at a friend or relative's house.

\section{Limitations of this study}

This study has several limitations.

NDSHS is subject to response bias from self-report. While there is some evidence demonstrating that selfreport of smoking in the home is moderately correlated to environmental and biomedical markers, ${ }^{30}$ it remains an inferior replacement for such measures. ${ }^{29}$ It is quite likely that home second-hand smoke exposure from this survey is therefore a conservative estimate.

In addition to respondents under-reporting what is clearly a socially undesirable behaviour (smoking indoors in a household with children), there have been several studies exploring what people actually mean when they talk about household smoking rules, that is, how people define (to themselves and others) 'smoke indoors' and 'smoke outdoors'. 31 These qualitative studies suggest that defining smoking outdoors might be a somewhat varied and fluctuating category, possibly contributing to further underestimation of home second-hand smoke exposure. At present, we do not know how defining home smoking status is played out in Australia, or if there are any rural/urban differences in this regard. Gaining an understanding of what people know about children's home second-hand smoke exposure, what is meant by smoking outside the home, and what their home smoking behaviour actually is in Australia would both illuminate the findings of this study and contribute to understanding how best to support people in either quitting or making their home smoke free.

A limited number of potential household-level confounders were available in this dataset and there are other important confounders at the household level, such as the ages of people in the household or the number of smokers in the household, which we were unable to control for. 
As our analysis was conducted at the household level, we did not use data on individuals and therefore did not control for potential individual-level confounders known to be associated with smoking prevalence, such as mental health. ${ }^{3}$ We did not control for Aboriginal status as although there were (very limited) data available, Aboriginal status is nonsensical as a household-level variable, though an important individual-level confounder, given that the smoking prevalence among Aboriginal Australians is more than twice that of non-Aboriginal Australians. ${ }^{32}$ SEIFA is an area-level variable. At the Census Collection District area (around 100 houses), this may not always apply to individual households.

\section{CONCLUSION}

The proportion of smoking households with children remains an important public health concern. The finding that rurality remains associated with smoking households when other factors such as SES are controlled for suggests that we need to understand more about the context of smoking in rural Australia to provide support targeted at rural households in quitting.

Significant numbers of children continue to be exposed to second-hand smoke in their homes and to suffering the lifelong consequences on their physical health, academic performance and their own smoking behaviour in the future. Socioeconomic disadvantage continues to be a significant driver of differences in children's home second-hand smoke exposure in rural compared to urban areas. Further efforts to support smokers who smoke inside their homes containing children to quit or avoid smoking in the home would benefit these children. Understanding the smoking rules and behaviours inside smoking households, and people's knowledge of children's home second-hand smoke exposure, should provide better evidence to support those efforts.

Acknowledgements The authors would like to thank Dr Coral Gartner for her advice about handling the weighting of the data, and Dr Judy Singer for her support with the background research for this study.

Contributors JML obtained, organised and analysed the data, and drafted and revised the manuscript. MEP designed the study, analysed the data and revised the manuscript. All authors have read and approved the final version of the manuscript.

Funding This research received no specific grant from any funding agency in the public, commercial or not-for-profit sectors.

Competing interests MEP receives research grant funding from Pfizer Australia, through an Australian Research Council Linkage Grant.

Provenance and peer review Not commissioned; externally peer reviewed.

Data sharing statement No additional data are available.

\section{REFERENCES}

1. Royal College of Physicians. Passive smoking and children. A report by the Tobacco Advisory Group. London, UK: Royal College of Physicians, 2010.

2. Jones LL, Atkinson O, Longman $\mathrm{J}$, et al. The motivators and barriers to a smoke-free home among disadvantaged caregivers: identifying the positive levers for change. Nicotine Tob Res 2011;13:479-86.
3. Scollo M, Winstanley M. Tobacco in Australia: facts and issues. Secondary tobacco in Australia: facts and issues, 2012. http://www. tobaccoinaustralia.org.au

4. Goldade K, Choi K, Bernat DH, et al. Multilevel predictors of smoking initiation among adolescents: findings from the Minnesota Adolescent Community Cohort (MACC) study. Prev Med 2012;54:242-6.

5. de Leeuw R, Engels R, Scholte R. Parental smoking and pretend smoking in young children. Tob Control 2010;19:201-5.

6. Jarvis MJ, Mindell J, Gilmore A, et al. Smoke-free homes in England: prevalence, trends and validation by cotinine in children. Tob Control 2009;18:491-5.

7. Johansson A, Hermansson G, Ludvigsson J. How should parents protect their children from environmental tobacco-smoke exposure in the home? Pediatrics 2004;113:e291-5.

8. Bottorff JL, Johnson JL, Carey $\mathrm{J}$, et al. A family affair: aboriginal women's efforts to limit second-hand smoke exposure at home. Can J Public Health 2010;101:32-5.

9. World Health Organization. WHO Framework Convention on Tobacco Control. Geneva: World Health Organization, 2003. World Health Organization. The World Health Report 2002.

10. Borland R, Mullins R, Trotter $\mathrm{L}$, et al. Trends in environmental tobacco smoke restrictions in the home in Victoria, Australia. Tob Control 1999;8:266-71.

11. Borland $\mathrm{R}$, Yong $\mathrm{H}$, Cummings $\mathrm{K}$, et al. Determinants and consequences of smoke-free homes: findings from the International Tobacco Control (ITC) Four Country Survey. Tob Control 2006;15 (Suppl 3):iii42-50.

12. Commonwealth Government of Australia. Draft for consultation of the National Tobacco Strategy 2012-2018. Secondary draft for consultation of the National Tobacco Strategy 2012-2018, 2012. http://www.nationaldrugstrategy.gov.au/internet/drugstrategy/ publishing.nst/Content/draft-nts-12-18-callsubs

13. Australian Institute of Health and Welfare. Rural, regional and remote health: indicators of health. Secondary rural, regional and remote health: indicators of health, 2005. http://www.aihw.gov.au/ publication-detail/?id=6442467718

14. Australian Bureau of Statistics. Tobacco smoking in Australia: a snapshot (4831.055.001). Secondary tobacco smoking in Australia: a snapshot (4831.055.001), 2006. http://www.abs.gov.au/ausstats/ abs@.nsf $/ \mathrm{mf} / 4831.0 .55 .001$

15. Gartner CE, Hall WD. Is the socioeconomic gap in childhood exposure to secondhand smoke widening or narrowing? Tob Control Published Online First: 30 March 2012. doi: 10.1136/ tobaccocontrol-2011-050297.

16. Bolte G, Fromme H. Socioeconomic determinants of children's environmental tobacco smoke exposure and family's home smoking policy. Eur J Public Health 2009;19:52-8.

17. Dent CW, Maher JE, Pizacani BA, et al. Secondhand smoke exposure in Alaskan households with children. Rural Remote Health 2010;10;1564.

18. Wiener RC. Secondhand and thirdhand tobacco smoke exposure in children living in states containing Appalachian counties: a surveillance. J Rural Trop Public Health 2011;10:111-18.

19. Xiao L, Yang $Y, L i ~ Q$, et al. Population-based survey of secondhand smoke exposure of children in China. Biomed Environ Sci 2010;23:430-6.

20. McMillen R, Breen J, Cosby AG. Rural-urban differences in the social climate surrounding environmental tobacco smoke: a report from the 2002 Social Climate Survey of Tobacco Control. J Rural Health 2006;20:7-16.

21. Roy Morgan Research. National Drug Strategy Household Survey 2010 Final Technical Report. Canberra: Australian Institute of Health and Welfare and Department of Health and Ageing, 2011.

22. Australian Bureau of Statistics. Australian Standard Geographical Classification (ASGC) Remoteness Structure (RA) Digital Boundaries, Australia, 2006 Secondary Australian Standard Geographical Classification (ASGC) Remoteness Structure (RA) Digital Boundaries, Australia, 2006 August 14 2006. http://www.abs. gov.au/AUSSTATS/abs@.nsf/DirClassManualsbyTopic/ 9A784FB979765947CA25738C0012C5BA

23. Australian Institute of Health and Welfare. Remoteness classifications. Secondary remoteness classifications. http://www. aihw.gov.au/rural-health-remoteness-classifications/

24. Kelly BJ, Stain HJ, Coleman C, et al. Mental health and well-being within rural communities: the Australian Rural Mental Health Study. Aust J Rural Health 2010;18:16-24.

25. Australian Institute of Health and Welfare. Australian Data ArchiveNational Drug Strategy Household Survey Questionnaire, 2010. Secondary Australian Data Archive-National Drug Strategy Household Survey Questionnaire, 2010. http://www.ada.edu.au/ social-science/01237 
26. Australian Bureau of Statistics. Australian Standard Geographical Classification (ASCG): socio-economic indexes for areas (SEIFA)_technical paper 2006. Secondary Australian Standard Geographical Classification (ASCG): socio-economic indexes for areas (SEIFA)technical paper 2006. http://www.abs.gov.au/ausstats/abs@.nsf/mf/ 2039.0.55.001

27. Siahpush M, Borland R, Scollo M. Factors associated with smoking cessation in a national sample of Australians. Nicotine Tob Res 2003;5:597-602.

28. Idris $\mathrm{BI}$, Giskes $\mathrm{K}$, Borrell $\mathrm{C}$, et al. Higher smoking prevalence in urban compared to non-urban areas: time trends in six European countries. Health Place 2007;13:702-12.
29. Daly JB, Wiggers JH, Considine RJ. Infant exposure to environmental tobacco smoke: a prevalence study in Australia. Aust N Z J Public Health 2001;25:132-7.

30. Rumchev K, Jamrozik K, Stick S, et al. How free of tobacco smoke are 'smoke-free' homes? Indoor Air 2008;18:202-8.

31. Robinson J, Kirkcaldy AJ. Disadvantaged mothers, young children and smoking in the home: mothers' use of space within their homes. Health Place 2007;13:894-903.

32. Hudson L, White A, Roseby R. Tobacco smoke exposure in hospitalised Aboriginal children in Central Australia. $J$ Paediatr Child Health 2009;45:224-7. 